\title{
Associação entre Poliarterite Nodosa e Síndrome Antifosfolípide: Relato de Caso e Revisão de Literatura
}

\section{Association between Polyarteritis Nodosa and Antiphospholipid Syndrome: Case Report and Literature Review}

\author{
Izaias Pereira da Costa $^{(1)}$, Fábio Augusto Moron de Andrade ${ }^{(2)}$, Alex Magno Coelho Horimoto ${ }^{(3)}$
}

\section{RESUMO}

Descrevemos aqui o caso de um homem de 37 anos com poliarterite nodosa (PAN) associada à síndrome antifosfolípide (SAF), que evoluiu com disfunção endotelial intensa, diversos aneurismas e oclusões arteriais, incluindo pseudo-aneurisma da artéria gastroduodenal. Realizamos uma revisão da literatura desta rara associação entre PAN e SAF.

Palavras-chave: poliarterite nodosa (PAN), síndrome antifosfolípide (SAF), disfunção endotelial, aneurismas.

\section{INTRODUÇÃO}

Poliarterite nodosa (PAN) é um protótipo de vasculite sistêmica necrosante que afeta vasos de médio calibre, com manifestações clínicas decorrentes de isquemia e infarto dos tecidos e órgãos afetados ${ }^{(1)}$. Foi descrita inicialmente por Adolf Kussmaul e Rudolf Maier em 1866 e atualmente é classificada entre as vasculites sistêmicas primárias, pela Conferência de Consenso de Chapel Hill em 1994(2).

Tem uma baixa prevalência na população, com incidência anual variando entre dois a nove casos por milhão de pessoas. Homens e mulheres podem ser acometidos, com ligeira predominância masculina e ocorre em todos os grupos étnicos ${ }^{(2)}$ em qualquer idade, porém é mais freqüente entre a quarta e sexta décadas ${ }^{(3)}$.

PAN clássica é uma vasculite determinada por múltiplos fatores, incluindo genéticos e ambientais. A associação

\begin{abstract}
We describe here the case of a 37 years old man with polyarteritis nodosa (PAN) associated to antiphospholipid syndrome (APS), that developed intense endothelial dysfunction, several aneurisms and arterial occlusions, including pseudo-aneurism of the gastricduodenal artery. We performed a literature review this rare association between PAN and APS.
\end{abstract}

Keywords: polyarteritis nodosa, antiphospholipid syndrome, endothelial dysfunction, aneurisms.

de infecção pelo vírus da hepatite $\mathrm{B}(\mathrm{HBV})$ e PAN foi inicialmente descrita em $1970^{(4)} \mathrm{e}$ atualmente se estima que a presença de anticorpos anti-HBs, é encontrada em uma freqüência variável de 10 a $54 \%$ de todos os casos de PAN $^{(5)}$. Esta vasculite também tem sido associada com vírus da imunodeficiência humana(HIV) e da hepatite C (HCV), além de neoplasias malignas hematológicas, especificamente tricoleucemia ${ }^{(6)}$.

$\mathrm{Na}$ análise histopatológica das lesões da PAN, observase alterações inflamatórias que invadem todas as túnicas arteriais, com acometimentos segmentares, alternando áreas lesionadas com áreas integras. Caracterizam-se também por serem observadas lesões em vários estágios de evolução, proporcionar redução da luz arterial por espessamento da íntima e formação conseqüente de trombos ${ }^{(7)}$.

A síndrome antifosfolípide (SAF) é caracterizada por uma vasculopatia trombótica, não-inflamatória, e está asso-

Serviço de Reumatologia da Universidade Federal de Mato Grosso do Sul (UFMS), Serviço de Cirurgia Vascular da Sociedade Beneficente de Campo Grande, Santa Casa, Campo Grande (MS). Recebido em 26/01/06. Aprovado, após revisão, em 02/06/06.

1. Médico Reumatologista, Mestre e Doutor em Reumatologia pela Faculdade de Medicina da Universidade de São Paulo (USP), Professor Adjunto de Clínica Médica da UFMS, Coordenador do Programa de Residência Médica em Reumatologia da UFMS.

2. Médico Cirurgião Vascular, Presidente da Sociedade Brasileira de Cirurgia Vascular, Regional Mato Grosso do Sul (MS), Coordenador do Programa de Residência Médica em Cirurgia Vascular da Sociedade Beneficente de Campo Grande - Santa Casa.

3. Médico Reumatologista, Mestrando em Ciências da Saúde pela UFMS, Preceptor Voluntário do Programa de Residência Médica em Reumatologia da UFMS. Endereço para correspondência: Dr. Alex Magno Coelho Horimoto, Rua Amazonas, 1494, apto. 802, Vila Gomes, CEP 79022-130, Campo Grande, MS, Brasil, telefone (67) 9982-2665 / 3326-5100, e-mail: amchor@ibest.com.br 
ciada à presença de anticorpos antifosfolípides ${ }^{(8)}$. Anticorpos antifosfolípides compreendem um grupo heterogêneo de auto-anticorpos, que incluem os anticorpos anticardiolipina, demonstrados por métodos imunológicos, e os anticoagulantes lúpicos, detectados por testes de coagulação ${ }^{(9)}$. Clinicamente, manifesta-se por tromboses venosas e/ou arteriais, além de complicações gestacionais ${ }^{(10)}$.

Anticorpos antifosfolípides são encontrados em várias condições auto-imunes, infecciosas e neoplásicas, sendo descritos raramente em associação com vasculites sistêmicas primárias ${ }^{(9)}$.

Pela raridade da associação entre PAN e a presença do anticorpo antifosfolípide e por estar provavelmente relacionada a pior prognóstico da vasculite, relatamos a ocorrência dessa associação em um paciente atendido em nosso Serviço e revisamos a literatura.

\section{RELATO DO CASO}

Trata-se de homem de 37 anos, com antecedente de cirurgia de troca de valva mitral por disfunção idiopática há 10 anos e em uso do anticoagulante varfarina sódica, com dose diária de $5 \mathrm{mg}$, mantendo Tempo de Atividade da Protrombina (TAP) com INR entre 2,5 a 3,5Ui. O mesmo já havia sido investigado em outro Serviço para febre reumática $(\mathrm{FR})$, porém não apresentava evidências clínicas de infecções ou de contato recente com o Estreptococo do grupo A de Lancefield, bem como laboratoriais, após diversas dosagens de antiestreptolisina O (ASLO) negativas, no soro. Além disso, não apresentava nenhum outro critério maior de Jones para diagnóstico de FR.

Há dois anos iniciou quadro progressivo de dor e claudicação intermitente em membro inferior esquerdo, decorrente de alterações isquêmicas. Realizado ecocolorDoppler e arteriografia, verificou-se estenose moderada em terço distal de artéria tibial anterior e crítica em terço distal de artéria tibial posterior, ambas em perna esquerda. Apresentou, na época, emagrecimento, dor e hipersensibilidade testicular, artralgia em grandes articulações (joelhos, tornozelos e punhos), adinamia e mialgia.

Evoluiu, após um ano, com claudicação em membro inferior direito, sendo internado devido piora da dor e frialdade em perna direita. Submetido à nova arteriografia, evidenciou-se estenose em terço proximal da artéria tibial posterior direita, com obstrução e pseudo-aneurisma, além das mesmas estenoses críticas em perna esquerda. A biópsia arterial mostrou trombo organizado e vasculite neutrofílica com restos leucocitários acometendo a parede vascular de forma segmentar.

Demais exames laboratoriais: TAP com INR: 3,23; VHS: $89 \mathrm{~mm}$ na $\mathrm{l}^{\text {a }}$ hora; mucoproteínas: $11,7 \mathrm{mg} / \mathrm{dl}$ (1,9-4,9); PCR: 48mg/l (até 6); FAN e fator reumatóide negativos; ANCA negativo; VDRL e anti-HIV negativos; anti-HCV negativo; anti $\mathrm{HBs}>1000 \mathrm{Ui} / \mathrm{ml}$ (até 10,0); anti $\mathrm{HBc}$ IgG reagente; anti $\mathrm{HBc}$ IgM negativo; HbsAg e HbeAg negativos.

Submetido à pulsoterapia com ciclofosfamida, uso de corticóides, vasodilatadores e anticoagulantes, houve estabilização do quadro. No entanto, durante a evolução, apresentou novos fenômenos vaso-oclusivos em MMII.

A pesquisa de anticardiolipina resultou-se em anticorpo da classe IgG positiva igual a $32 \mathrm{Ui} / \mathrm{ml}$ (até $20 \mathrm{Ui} / \mathrm{ml}$ ) e IgM negativo. Repetido após seis meses, mostrou a confirmação da presença do anticorpo e aumento na titulação (40Ui/ml). Com o diagnóstico de SAF, foi mantida varfarina sódica em dose diária de $5 \mathrm{mg}$ e o TAP com INR entre 2,5 a 3,5 .

Realizada nova arteriografia em seis meses, constatou-se a progressão da trombose com oclusão de artéria poplítea infrapatelar e reenchimento por colaterais das artérias tibiais (anterior e posterior) até terço médio da perna bilateralmente. Foi mantido o tratamento clínico com anticoagulação por duas semanas, porém o paciente evoluiu com quadro de dor epigástrica de forte intensidade, sendo diagnosticado pelo ecocolorDoppler, pseudoaneurisma de artéria gastro-duodenal, confirmado com arteriografia seletiva do tronco celíaco (Figuras l e 2). Realizou-se embolização com microcoils com sucesso (Figuras 3 e 4 ).

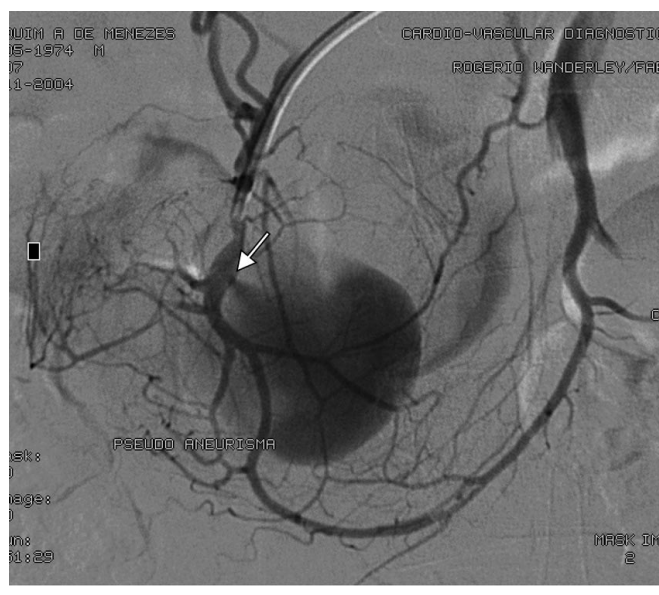

Figura 1 - Arteriografia digital com pseudo-aneurisma da artéria gastro-duodenal. 


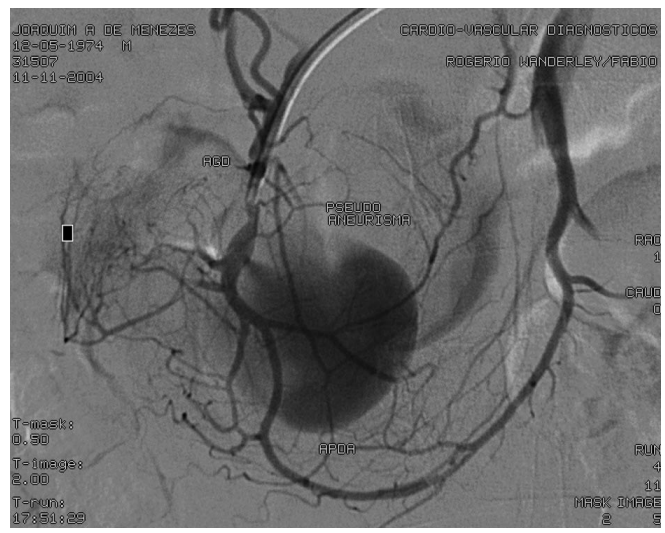

Figura 2 - Outra visão do pseudo-aneurisma através de arteriografia seletiva do tronco celíaco.

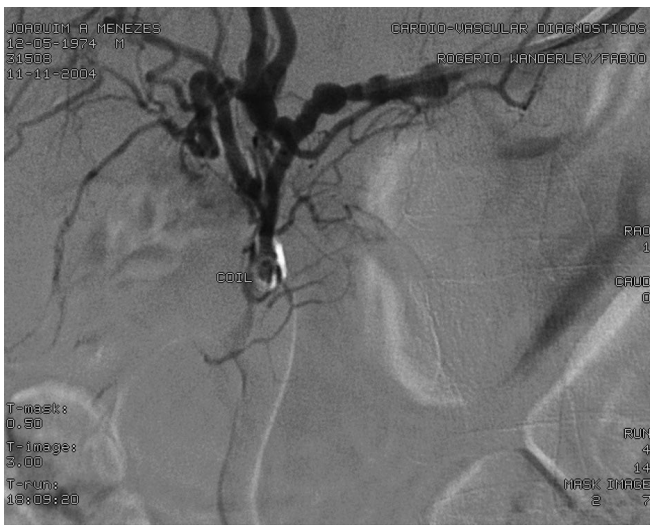

Figura 3 - Resolução do pseudo-aneurisma após embolização com microcoils.

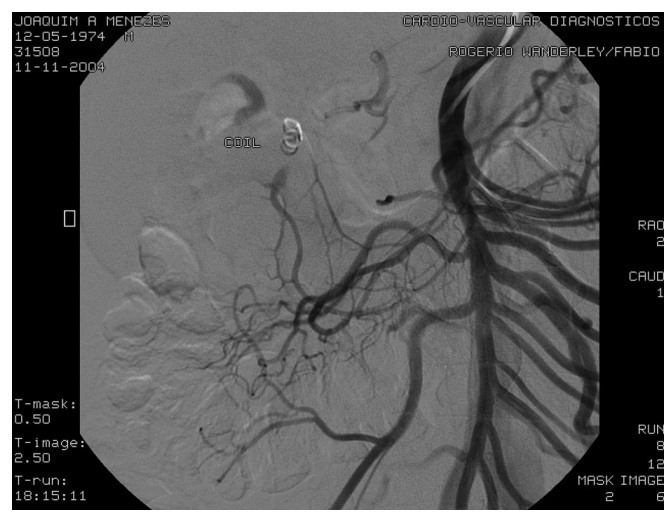

Figura 4 - Embolização do pseudo-aneurisma com microcoils.

Apresentou após uma semana, quadro de forte dor em fossa ilíaca esquerda, sendo que o ecocolorDoppler mostrou pseudo-aneurisma de artéria hipogástrica esquerda (Figura 5 ). Realizada nova cirurgia endovascular com implante de endoprótese revestida (Jostent graft 4,9 X $17 \mathrm{~mm}$ ) com sucesso (Figura 6).

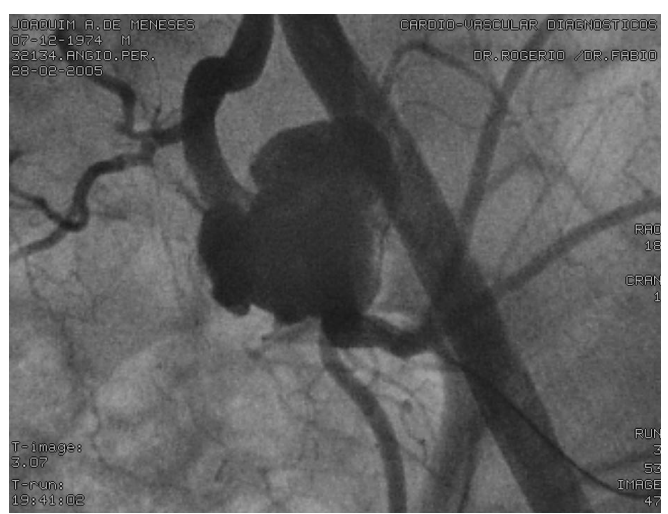

Figura 5 - Nova arteriografia com pseudo-aneurisma de artéria hipogástrica esquerda.

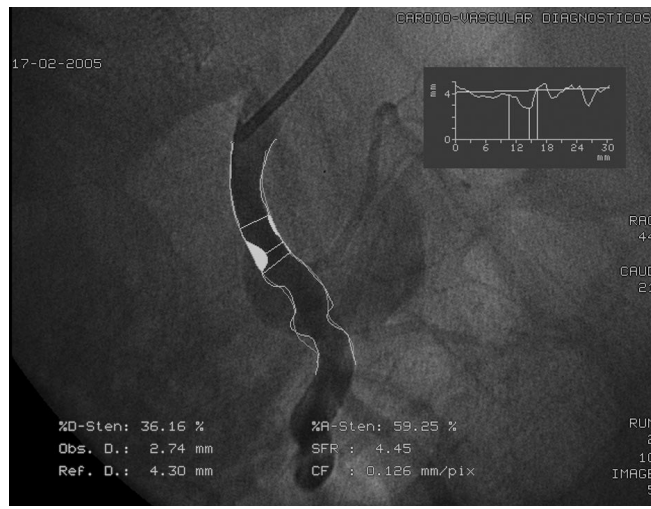

Figura 6 - Arteriografia com medidas para implante de endoprótese revestida (Jostent graft) por cirurgia endovascular.

Mesmo com anticoagulação em níveis adequados, com varfarina sódica na dose de $5 \mathrm{mg}$ ao dia associada ao ácido acetilsalicílico (AAS) na dose de $200 \mathrm{mg}$ ao dia e imunossupressão com pulsoterapia mensal com ciclofosfamida 0,6 $\mathrm{g} / \mathrm{m}^{2}$ de superfície corporal, continuava queixando-se de dores abdominais de forte intensidade, interpretada como claudicação mesentérica, sendo constatado na evolução outro pseudo-aneurisma em hipogástrica direita, através de tomografia computadorizada (TC) de abdome.

Finalmente, dois meses após o último procedimento cirúrgico, apresentou quadro de cefaléia súbita e intensa, acompanhada de hemianopsia homônima direita, vertigem, vômitos, dor abdominal e dificuldade à marcha.

Realizada TC de crânio foi compatível com ruptura de aneurisma em artéria cerebral posterior esquerda (Figura 7).

Durante internação no CTI foi necessária a suspensão do anticoagulante, devido ao quadro cerebral hemorrágico. No entanto, o paciente evoluiu com dor abdominal difusa e isquemia intestinal confirmada por arteriografia, que demonstrou 


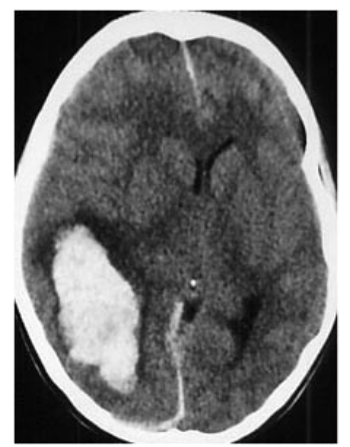

Figura 7 - TC de crânio compatível com ruptura de aneurisma em artéria cerebral posterior esquerda.

trombose do tronco celíaco, artérias mesentéricas superior e inferior, posteriormente evoluindo a óbito por choque sem resposta a drogas vasoativas. Não foi realizada necropsia por falta de autorização dos familiares.

\section{DISCUSSÃO}

Diversas síndromes envolvendo anticorpos antifosfolípides têm sido descritas na literatura. Embora manifestações clínicas variadas tenham sido bem documentadas, a fisiopatologia vascular em pacientes com estes anticorpos permanece pouco compreendida em alguns aspectos.

O dano vascular é freqüentemente descrito como uma vasculopatia trombótica, contudo existem alguns relatos que descrevem uma inflamação da parede vascular (vasculite) associada ${ }^{(11)}$. Goon et $a^{(12)}$ descreveram caso interessante de vasculite cutânea que tinha características de PAN e SAF, evoluindo com livedo reticular e gangrena digital sem envolvimento vascular em outros órgãos.

No entanto, Dongole e Foord ${ }^{(13)}$ ressaltaram, em 2000 , que pacientes com SAF, freqüentemente, se apresentam com manifestações trombóticas, mas existe um subgrupo de pacientes que desenvolvem aneurismas sem evidência de vasculite. Isso poderia representar parte de um espectro da própria SAF do que uma vasculite primária associada.

Outros autores como Dasgupta e Schoonjans ${ }^{(14,15)}$ reconheceram a existência real da associação entre PAN e SAF em pacientes descritos com aneurismas viscerais e biópsias compatíveis.

Finalmente, Praderio et a ${ }^{(16)}$ descreveram, em 1990, caso de paciente com diagnóstico documentado de PAN e evidência de anticorpo anticoagulante lúpico circulante. O paciente evoluiu com amputação dos dois primeiros dedos do pé devido à necrose isquêmica e só houve melhora com associação de tratamento imunossupressor e anticoagulante.

A associação entre PAN e SAF é rara e desperta grande interesse devido a potencial disfunção vascular grave, decorrente de mecanismos fisiopatogênicos distintos ${ }^{(17)}$. Não está claro se a presença de anticorpos antifosfolípides favorece o desenvolvimento de vasculites ou vice-versa.

Morelli et $a^{(18)} \mathrm{e}$ Valeyrie et al ${ }^{(19)}$ relataram outros casos de PAN com manifestações neurológicas e SAF concomitante.

Descreve-se ainda, em 2002, vasculite cerebral severa como primeira manifestação da PAN associada ao vírus da hepatite $\mathrm{B}^{(4,20)}$.

A patogênese da PAN está relacionada com vários fatores, incluindo linfócitos $\mathrm{T} \mathrm{CD}^{+}{ }^{+} \mathrm{CD}^{+}$, que desempenham um papel significativo na inflamação vascular. As células predominantes em biópsias nos infiltrados inflamatórios da parede vascular são linfócitos $\mathrm{T} \mathrm{CD}^{+}$e macrófagos. $\mathrm{O}$ recrutamento e ativação dos macrófagos são mediados por citocinas secretadas pelos linfócitos T CD4 $4^{+(2)}$.

São encontradas anormalidades quantitativas e qualitativas em citocinas nas síndromes vasculíticas, com aumento de IL-1, IL-2, IL-6 e TNF na fase ativa da doença. TNF e IL-1 são de particular importância por seus efeitos biológicos: efeito tóxico direto nas células endoteliais; aumento na expressão de moléculas de adesão nas células endoteliais; indução da produção de citocinas neutrófilo-atraentes.

As interações leucócito-endotélio via moléculas de adesão têm sido reconhecidas na patogênese da inflamação, incluindo vasculites. Níveis elevados de ICAM-1, VCAM1 e selectinas circulantes são encontrados em vasculites sistêmicas, incluindo PAN.

Por fim, anticorpos anticitoplasma de neutrófilos (ANCA) têm sido implicados como um fator etiológico em vasculites. Acredita-se que a liberação de proteinase $3 \mathrm{ou}$ mieloperoxidase, ambos alvos antigênicos, de grânulos primários de neutrófilos ou lisossomos de monócitos, resultam em ligação dos mesmos à parede endotelial com formação in situ de imunocomplexos.

A disfunção das células endoteliais (DCE) é comum em vasculites necrosantes sistêmicas como a PAN, levando à ativação direta das células e dano à parede vascular mediada pelo ANCA e possivelmente outros mediadores como$\mathrm{TNF}^{(21)}$. Descreve-se ainda DCE em pacientes com SAF, através da ligação dos anticorpos antifosfolípides à parede do vaso e que é ampliada pela expressão de moléculas de adesão, secreção de citocinas e alteração no metabolismo das prostaciclinas.

Dessa forma, consideramos que a presença do anticorpo 
antifosfolípide pode ter piorado o dano vascular provocado pela PAN neste paciente, via disfunção das células endoteliais, levando a aneurismas e oclusões vasculares múltiplas.

A ruptura de aneurismas é relativamente rara e representa uma condição reumatológica emergencial ${ }^{(22)}$, pode causar hemorragias viscerais principalmente de lesões complicadas em artérias mesentéricas e renais.

O comprometimento renal da SAF é raro e caracterizado por microangiopatia trombótica ${ }^{(23,24)}$. Na PAN, as manifestações renais ocorrem devido à vasculite de artérias renais, com aparecimento de múltiplos infartos e microaneurismas, além de hipertensão renovascular, insuficiência renal e proteinúria. No entanto, o paciente aqui relatado não apresentou este tipo de manifestação renal.

A PAN também pode se apresentar inicialmente com isquemia aguda de membros inferiores e evoluir com livedo reticular e gangrena de extremidades, assim como descrições de Fred et $a^{(25)}$, além de Héron et a ${ }^{(26)}$, ambos em 2003, e que estão em concordância com nosso relato.

Ressaltamos que este é o primeiro caso relatado de lesão vascular levando à pseudo-aneurisma da artéria gástricaduodenal e levantamos a possibilidade de que a valvulopatia mitral idiopática, apresentada pelo paciente, pode estar relacionada à SAF como uma das manifestações clínicas clássicas da síndrome, visto que o paciente nunca preencheu critérios diagnósticos para FR e, quando do diagnóstico da lesão mitral, estava acima da faixa etária habitual de desenvolvimento da mesma.

Futuros estudos são necessários para apoiar a hipótese da associação PAN-SAF desencadear DCE severa e determinar o regime terapêutico mais adequado nestes $\operatorname{casos}^{(27)}$.

Em relação ao tratamento cirúrgico atual, Stambo et $a^{(28)}$ realizaram a embolização com microcoils em múltiplos aneurismas hepáticos verdadeiros em um paciente com PAN diagnosticada e em tratamento e com altas doses de prednisona e citostático com excelentes resultados. Resultado semelhante foi obtido com aneurisma solitário ${ }^{(29)}$.

A doença em atividade aguda está geralmente associada com formação de aneurismas que, junto com a hipertensão devido a comprometimento renal ( $75 \%$ dos casos), aumenta o risco de ruptura significativamente ${ }^{(30)}$.

\section{CONCLUSÕES}

A presença do anticorpo antifosfolípide em um paciente com PAN, pode levar a um pior prognóstico, em decorrência da associação entre o seu potencial de desencadear fenômenos trombóticos e o processo inflamatório vascular da vasculite, o que pode agravar a vasculopatia mediada pela disfunção de células endoteliais que ocorre nas duas doenças, gerando casos potencialmente mais graves de lesão vascular. Podem, ainda, predispor a formação de pseudoaneurismas espontâneos pelas complicações do uso crônico de anticoagulantes e altas doses de corticóides, hipertensão renovascular e quadros de trombose de difícil tratamento devido à fragilidade inerente da parede da artéria comprometida por processo inflamatório.

Declaramos a inexistência de conflitos de interesse.

\section{REFERÊNCIAS}

1. Hughes LB, Bridges SL:Polyarteritis nodosa and microscopic polyangiitis: etiologic and diagnostic considerations. Curr Rheumatol Rep 4: 75-82, 2002.

2. Stone JH: Polyarteritis nodosa. JAMA 288 : 1632-9, 2002.

3. Mouthon L, Le Toumelin P, Andre MH et al: Polyarteritis nodosa and Churg-Strauss angiitis: characteristics and outcome in 38 patients over 65 years. Medicine (Baltimore) 81: 27-40, 2002.

4. Gullevin L, Lhote F, Cohen et al. Polyarteritis nodosa related to $\mathrm{HBV}$ infection. A prospective study with long-term observation of 41 patients. Medicine (Baltimore) 74:238-253, 1995.

5. Lau CF, Hui PK, Chan WM et al: Hepatitis B associated fulminant polyarteritis nodosa: successful treatment with pulse cyclophosphamide, prednisolone and lamivudine following emergency surgery. Eur J Gastroenterol Hepatol 14: 563-6, 2002.

6. Kuhn HM, Kromminga A, Flammann HT et al: P53 autoantibodies in patients with autoimmune diseases: a quantitative approach. Autoimmunity 31 : 229-35, 1999.

7. Ronco P, Verroust P, Mignon F et al. Immunopathological studies of polytarteritis nodosa and Wegener's granulomatosis: A report of 43 patients with 51 renal biopsies. Quart J Med 52:141-149; 1982.

8. Levine JS, Branch DW, Rauch J: The Antiphospholipid Syndrome. NEJM 346: 752-63, 2002.

9. Braham A, Safer L, Bdioni F et al: Les anticorps antiphospholipides en pathologie digestive. Presse Med 30: 1890-7, 2001.

10. Wilson WA, Gharavi AE, Koike T et al: International Consensus statement on preliminary classification criteria for definite 
antiphospholipid syndrome: report on an international workshop. Arthitis Rheum 42: 1309-11, 1999.

11. Norden DK, Ostrov BE, Shafritz AB et al: Vasculitis associated with antiphospholipid syndrome. Semin Arthitis Rheum 24 : 273-81, 1995.

12. Goon AT, Tay YK, Giam YC et al: An unusual case of cutaneous vasculitis. Ann Acad Med Singapore 29: 249-52, 2000.

13. Dongola NA, Foord KD: Angiographic features associated with antiphospholipid syndrome. Br J Radiol 73: 1215-8, 2000.

14. Dasgupta B, Almond MK, Tanqueray A: Polyarteritis nodosa and the antiphospholipid syndrome. Br J Rheumatol 36 : 1210-2, 1997.

15. Schoonjans R, Van Vlem B, Weyers $S$ et al: Polyarteritis nodosa and the antiphospholipid syndrome. Clin Rheumatol 15: 410-3, 1996.

16. Praderio L, D'Angelo A, Taccagni G et al: Association of lupus anticoagulant with polyarteritis nodosa: report of a case. Haematologica 75: 387-90, 1990.

17. Yoshiki T: Etiopathogenesis of necrotizing vasculitis. Intern Med 41: 39-40, 2002.

18. Morelli S, Perrone C, Paroli M: Recurrent cerebral infarctions in polyarteritis nodosa with circulating antiphospholipid antibodies and mitral valve disease. Lupus 7: 51-2, 1988.

19. Valeyrie L, Bachot N, Roujeau et al: Neurological manifestations of polyarteritis nodosa associated with the antiphospholipid syndrome. Ann Med Interne (Paris) 154: 479-82, 2003.

20. Semmo AN, Baumert TF, Kreisel W: Severe cerebral vasculitis as primary manifestation of hepatitis B-associated Polyarteritis nodosa. J Hepatol 37: 414- 6, 2002.

21. Filer AD, Gardner-Medwin JM, Thambyrajah J et al: Diffuse endothelial dysfunction is common to ANCA associated systemic vasculitis and Polyarteritis nodosa. Ann Rheum Dis
62: 162-7, 2003.

22. Kumar A, Marwaha V, Grover R: Emergencies in rheumatology. J Indian Med Assoc 101: 522-4, 2003.

23. Griffiths MH, Papadaki L, Neild GH: The renal pathology of primary antiphospholipid syndrome: a distinctive form of endothelial injury. QJM 93: 457-67, 2000.

24. Rollino C, Mazzucco G, Boero R et al: Is it possible to diagnose primary anti phospholipid syndrome (PAPS) on the basis of renal thrombotic microangiopathy (PAPS nephropathy) in the absence of other thrombotic process? Ren Fail 25: 1043-9, 2003.

25. Fred HL, Serna JH, Mc Donald GA et al: Images in cardiovascular medicine: Polyarteritis nodosa inducing symmetric peripheral gangrene. Circulation 107: 2870, 2003.

26. Héron E, Fiessinger JN, Guillevin L: Polyarteritis nodosa presenting as acute leg ischemia. J Rheumatol 30: 1344-6, 2003.

27. Guillevin L, Cohen P, Mahr A et al: Treatment of Polyarteritis nodosa and microscopic polyangiitis with poor prognosis factors: a prospective trial comparing glucocorticoids and six or twelve cyclophosphamide pulses in sixty-five patients. Arthritis Rheum $49: 93-100,2003$.

28. Stambo G, Guiney MJ, Cannella XF, German BF: Coilembolization of multiple hepatic artery aneurisms in a patient with undiagnosed polyarteritis nodosa. Journal of Vascular Surgery 39: 1122-4, 2004.

29. Herskowitz MM, Flyer MA, Sclafani SJ: Percutaneous transhepatic coil embolization of a ruptured intrahepatic aneurysm in poliarteritis nodosa. Cardiovasc Intervent Radiol 16: 254-6, 1993.

30. Choy CWK, Smith PA, Frazer C, Jeffrey GP: Ruptured hepatic artery aneurysm in poliarteritis nodosa: a case report and literature review. Aust NZ J Surg 67: 906-8, 1997. 